\title{
Papers
}

\section{Alcohol drinking in middle age and subsequent risk of mild cognitive impairment and dementia in old age: a prospective population based study}

Tiia Anttila, Eeva-Liisa Helkala, Matti Viitanen, Ingemar Kåreholt, Laura Fratiglioni, Bengt Winblad, Hilkka Soininen, Jaakko Tuomilehto, Aulikki Nissinen, Miia Kivipelto

\begin{abstract}
Objective To evaluate the relation between midlife alcohol consumption and mild cognitive impairment and dementia in old age, and the possible modification of this relation by apolipoprotein E.

Design Prospective, population based study.

Setting Populations of Kuopio and Joensuu, eastern Finland. Participants Of 1464 men and women aged 65-79 years randomly selected from population based samples studied in 1972 or $1977,1018(70 \%)$ were re-examined in 1998 (after an average follow up of 23 years).
\end{abstract}

Main outcome measures Mild cognitive impairment and dementia in old age.

Results Participants who drank no alcohol at midlife and those who drank alcohol frequently were both twice as likely to have mild cognitive impairment in old age as those participants who drank alcohol infrequently. The risk of dementia related to alcohol drinking was modified by the presence of the apolipoprotein e4 allele. The carriers of apolipoprotein e4 had an increased risk of dementia with increasing alcohol consumption: compared with non-carriers who never drank, the odds ratio for carriers who never drank was 0.6, for infrequent drinkers it was 2.3 , and for frequent drinkers was 3.6 (the overall interaction term "drinking

frequency*apolipoprotein e4" was significant $(\mathrm{P}=0.04)$, as were the interactions "infrequent drinking*apolipoprotein e4" $(\mathrm{P}=0.02)$ and "frequent drinking*apolipoprotein e4" $(\mathrm{P}=0.03))$. Non-carriers of apolipoprotein $\mathrm{e} 4$ had similar odds ratios for dementia irrespective of alcohol consumption.

Conclusion Alcohol drinking in middle age showed a U shaped relation with risk of mild cognitive impairment in old age. Risk of dementia increased with increasing alcohol consumption only in those individuals carrying the apolipoprotein 44 allele.

\section{Introduction}

Alcohol drinking has been proposed as a possible risk factor for cognitive impairment and dementia, though understanding the association has proved difficult. Some studies have found no association between alcohol drinking and cognitive impairment ${ }^{1-3}$ or Alzheimer's disease, ${ }^{45}$ whereas others have found an association between heavy drinking and increased risk of dementia. ${ }^{7}$ Some have claimed there is a J or U shaped relation between alcohol drinking and cognitive impairment $t^{8-10}$ or dementia ${ }^{11-14}$; that is, light to moderate alcohol drinking might have a protective effect compared with total abstention and heavy drinking. The apolipoprotein e4 allele, which is the only genetic risk factor for dementia with an established risk for the general population, may modify the effects of alcohol on the risk of developing cognitive impairment ${ }^{15}{ }^{16}$ or dementia. ${ }^{12}{ }^{17}$ However, the observed pattern of the effect modification has been different across the studies.

Earlier studies of alcohol drinking and cognition defined cognitive impairment in terms of performance in a variety of neuropsychological tests and did not validate it by any clinical or diagnostic concepts such as the criteria defining mild cognitive impairment. ${ }^{18}$ Mild cognitive impairment is considered to be a clinically identifiable entity and to carry a high risk for progression to dementia. ${ }^{19}{ }^{20}$ Studies of alcohol drinking and dementia have mainly been either cross sectional or carried out in elderly cohorts with relatively short follow up times, making them prone to biases caused by subclinical dementia and other factors.

In order to clarify the role of alcohol in the development of dementia, one needs prospective studies with data on earlier alcohol drinking and long follow up. Given that midlife risk factors contribute to the onset of the dementing disease processes, ${ }^{21}$ we investigated whether alcohol drinking in middle age could influence the subsequent risk of dementia. The aim of this study was to evaluate the association between midlife alcohol drinking and the risk of mild cognitive impairment and dementia in old age. We also investigated whether the apolipoprotein e4 allele modifies this association.

\section{Participants and methods}

Participants

We selected our study participants from those in the cardiovascular risk factors, aging and dementia (CAIDE) study, which is described in detail elsewhere. ${ }^{22}$ The participants were randomly selected from population based samples in two areas in eastern Finland. They were enrolled in 1972, 1977, 1982, and 1987 within the framework of the North Karelia project and the Finnish part of the monitoring trends and determinants in cardiovascular disease (FINMONICA) study. ${ }^{23-25}$ Participation rates in these surveys ranged from $82 \%$ to $90 \%$.

A random sample of 2000 individuals aged 65-79 years in 1997 who were still alive and living in the study area were invited for re-examination for the CAIDE study, and altogether 1449 $(72.5 \%)$ were investigated in 1998 . For the current study, we used the subsamples who were first enrolled in 1972 and 1977. Of 
these 1464 people, $1018(70 \%)$ participated. These 632 women $(62 \%)$ and 386 men (38\%) had a mean (SD) age of $48.3(4.8)$ years at the time of their midlife examination and 71.7 (4.1) years at their follow up examination in 1998. We obtained written informed consent from all participants in 1998.

\section{Surveys}

The methods used in the baseline (midlife) survey were standardised to follow international recommendations. ${ }^{25}$ In brief, the survey included a self administered questionnaire on health behaviour, health status, medical history (including specific inquiry about any cerebrovascular or cardiovascular events and conditions diagnosed by a physician), and socioeconomic factors. Nurses trained for the survey went through the questionnaires to ensure that they were fully completed. Participants' blood pressure, height, and weight were measured, and body mass index (weight $(\mathrm{kg}) /\left(\right.$ height $(\mathrm{m})^{2}$ ) was calculated. A venous blood specimen was taken to determine serum cholesterol, ${ }^{25}$ and apolipoprotein $\mathrm{E}$ genotypes were analysed by means of the polymerase chain reaction and HhaI digestion as described by Tsukamoto. ${ }^{26}$

\section{Alcohol consumption}

The 1972 and 1977 questionnaire asked for details of the overall frequency of alcohol drinking. For the present study, we classified people as those who never drank alcohol, those who drank "infrequently" (less than once a month), and those who drank "frequently" (several times a month). In the follow up examination in 1998, we asked about alcohol consumption in more detail, from which we derived the comparable categories with the earlier survey.

\section{Cognitive assessments}

The diagnostic procedures of the present study are described in greater detail elsewhere. ${ }^{22}$ In brief, cognitive function was assessed in 1998 with a three step protocol for diagnosing dementia and mild cognitive impairment. Participants who scored $\leq 24$ on the mini-mental state examination ${ }^{27}$ were selected for further examination.

The diagnostic criteria used for diagnosing mild cognitive impairment were those proposed by the Mayo Clinic Alzheimer Disease Research Center. ${ }^{28}{ }^{29}$ These included (a) memory complaint by patient, family, or physician; $(b)$ normal activities of daily living; $(c)$ normal global cognitive function; $(d)$ objective impairment of memory or one other area of cognitive function, as shown by scores $>1.5$ standard deviations below the age appropriate mean; $(e)$ clinical dementia rating score of 0.5 ; and $(f)$ not demented.

The diagnosis of dementia was based on the Diagnostic and Statistical Manual of Mental Disorders, fourth edition, with Alzheimer's disease being diagnosed according to the criteria of the National Institute of Neurological and Communicative Disorders and Stroke and the Alzheimer's Disease and Related Disorders Association. ${ }^{30}$

Altogether $61(5.8 \%)$ participants met the criteria for mild cognitive impairment, and $48(4.6 \%)$ met the criteria for dementia, of whom $37(77 \%)$ had Alzheimer's disease. The total number of dementia cases increased to 88 (6.0\% of the population) when we included information regarding the non-participants from their patient records at the local hospitals and primary healthcare centres. The people who did not fulfil the criteria for mild cognitive impairment or dementia formed the control group for our study.

\section{Statistical analyses}

We analysed the data using SPSS for Windows, version 11.0 (SPSS, Chicago, IL). We used analyses of variance and the $\chi^{2}$ test to test the differences between the alcohol consumption groups (never, infrequently, or frequently). We investigated the association between midlife alcohol and subsequent mild cognitive impairment and dementia by means of multiple logistic regression analyses, calculating odds ratios with $95 \%$ confidence intervals. Since some studies, as well as our preliminary analyses, indicated that there was a J or $\mathrm{U}$ shaped relation between alcohol drinking and mild cognitive impairment and dementia, we used infrequent drinkers as our reference group.

All analyses were adjusted for age, sex, and education. Further analyses controlled also for the variables related with midlife alcohol drinking frequency (midlife body mass index, total cholesterol concentration, smoking status (smoker or nonsmoker), and follow up time) and for other vascular factors (midlife systolic and diastolic blood pressure, history of myocardial infarction and stroke at follow up).

We used stratified analyses to evaluate how different frequencies of alcohol drinking affected the risk of mild cognitive impairment or dementia separately in carriers and non-carriers of the apolipoprotein e 4 allele. We then tested the putative interaction between alcohol drinking and carrier status for apolipoprotein e4 (e4 carrier/non-carrier) by adding the interaction term for "alcohol drinking*apolipoprotein e4" into the logistic regression model. From this model we calculated odds ratios for each "alcohol drinking*apolipoprotein e4" category.

We tested the interactions between alcohol drinking and sex in a similar manner. The level of significance was $\mathrm{P} \leq 0.05$ in all presented results.

\section{Results}

Characteristics of the participants according to their midlife alcohol drinking

About $30 \%$ of the participants never drank alcohol, $40 \%$ drank it infrequently, and 30\% drank frequently (table 1). Among the frequent drinkers, about $68 \%$ drank alcohol once or twice a month, $24 \%$ once a week, about $8 \%$ twice a week, and $1 \%$ daily. Among the infrequent drinkers, more than $40 \%$ drank alcohol twice a year or less frequently, and about $60 \%$ reported drinking three to six times a year.

Non-drinkers were the oldest group at the follow up examination, and frequent drinkers were the youngest (table 1). Non-drinkers were less educated than the other two groups. Women were more likely to be non-drinkers or infrequent drinkers, whereas men were more likely to be frequent drinkers. Smoking was most common among frequent drinkers. Body mass index and total cholesterol concentration tended to be lower among infrequent drinkers than in the other two groups. The proportion of people with a history of myocardial infarction and stroke did not differ significantly according to drinking frequency either at middle age (a few cases only, data not shown) or old age. Mild cognitive impairment in old age was most common among non-drinkers and least common in infrequent drinkers. The frequency of old age dementia did not significantly differ with drinking frequency (table 1).

\section{Alcohol consumption in middle age and cognitive impairment in old age}

Participants who reported never drinking alcohol and those who reported drinking alcohol frequently were both more than twice as likely to have mild cognitive impairment in old age than those 
Table 1 Sociodemographic and clinical characteristics of 1018 participants from eastern Finland according to their midlife alcohol consumption. Values are means (standard deviations) unless stated otherwise

\begin{tabular}{|c|c|c|c|c|}
\hline \multirow[b]{2}{*}{ Characteristics } & \multicolumn{3}{|c|}{ Alcohol drinking } & \multirow[b]{2}{*}{$P$ value* } \\
\hline & Never $(n=300)$ & Infrequent ( $\mathrm{n}=423$ ) & Frequent $(\mathrm{n}=295)$ & \\
\hline Age at baseline (years) & $48.4(5.0)$ & $47.8(4.9)$ & $47.7(4.5)$ & 0.11 \\
\hline Age at follow up (years) & $72.3(4.2)$ & $71.4(4.1)$ & $70.8(3.9)$ & $<0.01$ \\
\hline Follow up time (years) & $23.8(2.5)$ & $23.6(2.5)$ & $23.1(2.4)$ & $<0.01$ \\
\hline Time in education (years) & $8.2(3.3)$ & $8.8(3.3)$ & $8.9(3.7)$ & 0.02 \\
\hline No (\%) of men:women & 50 (17):250 (83) & $149(35): 274(65)$ & 187 (63): $108(37)$ & $<0.01 \dagger$ \\
\hline No (\%) of apolipoprotein e4 carriers & $112 / 291$ (38) & $145 / 411(35)$ & $102 / 289(35)$ & $0.63 \dagger$ \\
\hline \multicolumn{5}{|l|}{ Midlife measurements (baseline) } \\
\hline Systolic blood pressure (mm Hg) & $144.3(20.5)$ & $142.7(19.6)$ & $143.6(18.2)$ & 0.52 \\
\hline Diastolic blood pressure (mm Hg) & $90.2(10.0)$ & $89.6(10.9)$ & $90.9(10.9)$ & 0.26 \\
\hline $\begin{array}{l}\text { Total serum cholesterol concentration } \\
(\mathrm{mmol} / \mathrm{l})\end{array}$ & $6.9(1.3)$ & $6.7(1.2)$ & $6.8(1.1)$ & 0.09 \\
\hline Body mass index $\left(\mathrm{kg} / \mathrm{m}^{2}\right)$ & $26.6(3.7)$ & $26.0(3.6)$ & $26.4(3.3)$ & 0.10 \\
\hline No $(\%)$ of smokers & $54(18)$ & $193(46)$ & $204(69.2)$ & $<0.01 \dagger$ \\
\hline \multicolumn{5}{|l|}{ Old age measurements (follow up) } \\
\hline No (\%) who have had myocardial infarction & $41 / 295(14)$ & $60 / 414(14)$ & $54 / 292(18)$ & $0.24 \dagger$ \\
\hline No (\%) who have had stroke & $22 / 289(8)$ & $32 / 410(8)$ & $18 / 286(6)$ & $0.71 \dagger$ \\
\hline \multicolumn{5}{|l|}{ Results of cognitive assessment: } \\
\hline No (\%) with no impairment (controls) & $261(87)$ & $391(92)$ & $257(87)$ & $0.02 \dagger$ \\
\hline No (\%) with mild cognitive impairment & $25(8)$ & $15(4)$ & $21(7)$ & $0.02 \dagger$ \\
\hline No (\%) with dementia & $14(5)$ & $17(4)$ & $17(6)$ & $0.56 \dagger$ \\
\hline
\end{tabular}

${ }^{\star}$ Analysis of variance used unless otherwise indicated. $\dagger \chi^{2}$ test used.

who drank infrequently, even after we controlled for age, sex, and years of education (table 2). The results remained essentially the same when we repeated the analyses with additional adjustment for factors related to alcohol drinking or increased risk of dementia, including follow up time, smoking, total serum cholesterol concentration, body mass index, systolic and diastolic blood pressure, and vascular diseases in old age. The risk of dementia did not significantly differ between alcohol drinking categories (table 2). Adding apolipoprotein $\mathrm{E}$ genotype to this second model provided only slightly different odds ratios for dementia (non-drinkers 0.81 (95\% confidence interval 0.34 to 1.93 ), infrequent drinkers 1 (reference), frequent drinkers 1.57 (0.70 to 3.50)) and for mild cognitive impairment (non-drinkers 2.03 (0.95 to 4.34), infrequent drinkers 1 , frequent drinkers 2.60 (1.19 to 5.69$))$

\section{Effect of apolipoprotein e4 allele}

To investigate whether apolipoprotein $\mathrm{E}$ genotype modified the effect of midlife alcohol drinking on the risk of dementia in old age, we performed analyses stratified by apolipoprotein e4 carrier status. Because our results indicated that the risk of dementia increased with increasing alcohol drinking frequency (see table 2), we used the group who never drank as the reference

Table 2 Results of multiple logistic regression analyses showing influence of participants' midlife alcohol consumption on their cognitive status in old age. Values are odds ratios ( $95 \%$ confidence intervals)

\begin{tabular}{|c|c|c|c|}
\hline \multirow[b]{2}{*}{ Cognitive status } & \multicolumn{3}{|c|}{ Alcohol drinking } \\
\hline & Never $(n=300)$ & Infrequent $(n=423)$ & Frequent $(n=295)$ \\
\hline \multicolumn{4}{|l|}{ Dementia: } \\
\hline Model $1^{*}$ & 0.88 (0.42 to 1.83$)$ & 1 (reference) & 1.45 (0.72 to 3.01$)$ \\
\hline Model $2 \dagger$ & 0.91 (0.39 to 2.14$)$ & 1 & $1.44(0.66$ to 3.15$)$ \\
\hline \multicolumn{4}{|l|}{$\begin{array}{l}\text { Mild cognitive } \\
\text { impairment: }\end{array}$} \\
\hline Model $1^{*}$ & 2.08 (1.05 to 4.13$)$ & 1 & 2.34 (1.15 to 4.77$)$ \\
\hline Model $2 \dagger$ & 2.15 (1.01 to 4.59 ) & 1 & 2.57 (1.19 to 5.52$)$ \\
\hline
\end{tabular}

*Analyses adjusted for age, sex, and education.

$\dagger$ Analyses adjusted for age, sex, education, follow up time, body mass index, total serum

cholesterol, systolic blood pressure, diastolic blood pressure, smoking, history of myocardial infarction, and history of stroke. group. Among carriers of the apolipoprotein e4 allele, the risk of dementia increased with increasing alcohol consumption after all the adjustments (odds ratio for never drinking 1 (reference), for infrequent drinking 4.08 (95\% confidence interval 0.98 to 16.91), and for frequent drinking 7.07 (1.37 to 36.60)). Among non-carriers of apolipoprotein e4, the risk of dementia did not change significantly with alcohol drinking categories (odds ratio for never drinking 1 , for infrequent drinking 0.45 (0.13 to 1.60), and for frequent drinking 0.75 (0.19 to 2.99$)$ ).

To detect the combined effect of apolipoprotein $\mathrm{E}$ genotype and alcohol drinking in relation to dementia, we introduced the interaction term "drinking frequency*apolipoprotein e4" into the logistic regression model together with apolipoprotein e4, drinking frequency, and other covariates. This showed a significant interaction between apolipoprotein $\mathrm{E}$ and alcohol drinking frequency, with the overall interaction term being significant $(\mathrm{P}=0.04)$. The interaction terms "infrequent drinking*apolipoprotein e4" and "frequent drinking*apolipoprotein $\mathrm{e} 4$ " were also significant $(\mathrm{P}=0.02$ and $\mathrm{P}=0.03$, respectively). The figure shows the odds ratios derived from this model for each of the six possible combinations between apolipoprotein $\mathrm{e} 4$ and drinking frequency. Compared with the participants who never drank and did not carry the apolipoprotein e4 allele, e4 carriers who drank infrequently were 2.3 times more likely to develop dementia, and carriers who drank frequently were 3.6 times more likely. However, the risk of dementia for e4 carriers who never drank was not different from that for non-carriers who never drank. Among the non-carriers, there was no association between alcohol drinking and the risk of dementia.

The effect modification by apolipoprotein e 4 for the association between alcohol drinking and mild cognitive impairment in the interaction model did not reach significance $(\mathrm{P}=0.54$ for the overall interaction term, $\mathrm{P}=0.39$ for "never drinking*apolipoprotein e4," and $\mathrm{P}=1.00$ for "frequent drinking*apolipoprotein e4," with non-carriers of apolipoprotein e4 who drank infrequently as the reference group).

As mild cognitive impairment and dementia can be seen as points on the same cognitive continuum, we analysed how apoli- 


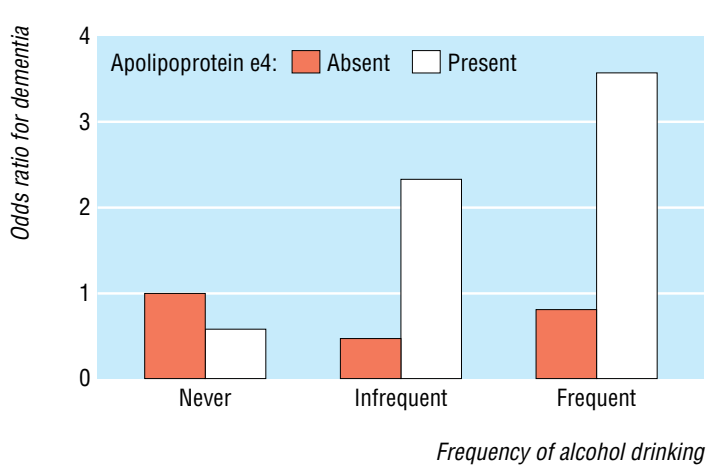

Combined effect of midlife alcohol drinking and apolipoprotein e4 allele on risk of dementia in old age. Values are odds ratios from one logistic regression analysis with alcohol drinking frequency, apolipoprotein e4, and the interaction term "drinking frequency*apolipoprotein e4" in the model and fully adjusted (see methods). Non-carriers of apolipoprotein e4 who never drank were the reference group. P value for the overall interaction "drinking frequency*apolipoprotein e4" is 0.04 . The odds ratio for the interaction term "infrequent drinking *apolipoprotein e4" is $8.52(\mathrm{P}=0.02)$ and for "frequent drinking *apolipoprotein e4" is 7.64 ( $\mathrm{P}=0.03$ )

poprotein e4 modified the association between alcohol drinking and cognitive impairment (including both mild cognitive impairment and dementia). We found similar results as those seen for dementia alone: there was a significantly increased risk among e4 carriers who drank alcohol, more so among frequent drinkers than infrequent drinkers. For the entire interaction between alcohol drinking and apolipoprotein $\mathrm{e}$, the $\mathrm{P}$ value was 0.045. The odds ratio for the interaction term "infrequent drinking*apolipoprotein $\mathrm{e} 4$ " was $3.87(\mathrm{P}=0.02)$, and for "frequent drinking*apolipoprotein e4" it was $3.00(\mathrm{P}=0.05)$.

Among men, frequent alcohol drinking was related to an increased risk of mild cognitive impairment (odds ratio $=5.03$, $\mathrm{P}=0.02$ ) compared with infrequent drinking, but never drinking did not significantly increase the risk (odds ratio $=3.84, \mathrm{P}=0.12$ ). Among women, neither frequent drinking nor never drinking was significantly associated with an increased risk of mild cognitive impairment (odds ratios $1.63(\mathrm{P}=0.43)$ and $1.41(\mathrm{P}=0.44)$ respectively). Nevertheless, there was no interaction between sex and alcohol drinking frequency for the risk of mild cognitive impairment. Alcohol drinking was not significantly related to risk of dementia in the analyses when we included sex interactions (data not shown).

The results concerning the risk of dementia remained unchanged when we repeated the analyses with the dementia diagnoses derived from the patient registries also taken into account.

\section{Alcohol consumption in old age and cognitive impairment} Alcohol consumption in old age was in agreement with midlife consumption $(\kappa=0.36, \mathrm{P}<0.01)$. In the analyses of the association between old age drinking and the prevalence of mild cognitive impairment, we found again that non-drinkers more often had mild cognitive impairment than did infrequent drinkers, even after we adjusted for age, education, and sex (data not shown). However, frequent alcohol drinking was no longer associated with mild cognitive impairment. Old age drinking was not associated with dementia. Further adjustments did not change these results. The apolipoprotein e 4 allele did not significantly modify the association between old age drinking and mild cognitive impairment or dementia.

\section{Non-participants}

Non-participants at the follow up visit (1998) were, at the midlife assessment, older than the participants (mean age $49.1 v 48.0$ years, $\mathrm{P}=0.01)$; had spent less time in education (7.7 $v 8.7$ years, $\mathrm{P}<0.01)$; and had higher systolic blood pressure (148.9 $v 143.4$ $\mathrm{mm} \mathrm{Hg}, \mathrm{P}<0.01)$, diastolic blood pressure $(92.3$ v $90.1 \mathrm{~mm} \mathrm{Hg}$,
$\mathrm{P}<0.01)$, total serum cholesterol concentration $(7.0$ v 6.8 $\mathrm{mmol} / \mathrm{l}, \mathrm{P}<0.01)$, and body mass index (26.9 $v 26.3, \mathrm{P}<0.01)$. They also had dementia in old age (data from patient records) more often than the participants $(8.0 \%$ v $5.1 \%, \mathrm{P}=0.03)$. However, their midlife alcohol consumption did not differ significantly from that of the participants (non-drinkers 34.8\% v $29.5 \%$, infrequent drinkers $37.9 \% v 41.6 \%$, and frequent drinkers $27.4 \% \cup 29.0 \%, \mathrm{P}=0.13)$, and the prevalence of smoking was similar $(47.7 \%$ v 44.3\%, $\mathrm{P}=0.23)$. The sex distribution of non-participants was no different from that of participants $(64.3 \%$ v $62.1 \%$ women, $\mathrm{P}=0.42)$.

\section{Discussion}

This study showed that midlife alcohol drinking was related to the risk of mild cognitive impairment in old age in a $U$ shaped manner, with both non-drinkers and frequent drinkers having a higher risk than infrequent drinkers. In contrast, no U shaped relation was found for dementia. However, the apolipoprotein $\mathrm{E}$ genotype seemed to modify the relation, with risk of old age dementia increasing with increasing midlife alcohol consumption only among carriers of the apolipoprotein e4 allele.

On the basis of these findings, we suggest that the path from normal cognitive function to mild cognitive impairment is influenced by environmental factors, such as frequent alcohol drinking. However, a combined effect of detrimental environmental factors (frequent drinking in this case) and genetic susceptibility factors, such as apolipoprotein e4, may be needed for cognitive impairment to progress into dementia.

\section{Effects of alcohol consumption}

Recent studies have detected a U shaped relation between alcohol consumption and various health outcomes, including coronary heart disease, stroke, dementia, and cognitive impairment. ${ }^{31}$ To our knowledge, our study is the first to show a similar association to mild cognitive impairment. Previously, associations between alcohol drinking and cognitive impairment were assessed according to various psychometric based definitions of cognitive impairment. The different measures used mean it is difficult to compare different studies and to determine the relevance of cognitive impairment in participants' lives. The concept of mild cognitive impairment takes into account both psychometric and clinical aspects, and so mild cognitive impairment may be considered to signify a clinically relevant entity itself; it has also been suggested to represent a high risk state for dementia. 
Interestingly, the $\mathrm{U}$ shaped relation we found between alcohol consumption and mild cognitive impairment has also been found in other populations with respect to their different drinking patterns and different alcohol levels. ${ }^{8-14}$ It is possible that moderate lifestyles in general, which obviously vary according to different cultural environments, protect from cognitive deterioration. Thus, it may not be the direct effect of alcohol or specific substances in alcoholic drinks that provide the protection, but moderate alcohol drinking may be an indicator of a complex set of favourable social and lifestyle factors. Some studies have proposed that the protective effect of alcohol is due to specific substances, such as flavonoids, in wine. ${ }^{32}{ }^{33}$ This is unlikely to be the reason for our results, since the most typical alcoholic drinks in Finland at the time of our study were beer and distilled liquor.

In the Rotterdam study ${ }^{12}$ the protective effect of alcohol drinking was found mainly for vascular dementia, and the authors suggested that moderate alcohol intake might protect against dementia via a reduction in vascular risk factors. In our study, most of the demented subjects had Alzheimer's disease, and we could not analyse the effect of alcohol consumption on vascular dementia separately because of the small number of cases. In our analyses, however, we controlled for vascular risk factors and vascular diseases, but the protective effect of infrequent drinking against mild cognitive impairment persisted.

\section{Effects of apolipoprotein $\mathrm{E}$ genotype}

Genetic susceptibility seems to modify the effect of alcohol on risk of dementia. This may explain why we did not find pronounced associations between alcohol drinking and risk of dementia until we took into account the effect of apolipoprotein $\mathrm{E}$ genotype. Our results of the modifying effect of the apolipoprotein e4 allele agree with those from a US study with regard to dementia ${ }^{17}$ and from a French study for cognitive test performance. ${ }^{16}$ One possible explanation could be that people with the e4 allele have less effective neural repair mechanisms, ${ }^{34}$ and thus they would be more susceptible to the deleterious effects of alcohol.

The Rotterdam study also found a possible interaction between alcohol and apolipoprotein e4, but in this case an increase in the protective effect of light to moderate alcohol consumption against dementia. ${ }^{12}$ The difference between these results and ours may be attributable to the different patterns of alcohol drinking in these two European countries. Typically, this birth cohort of Finns, particularly women, had a relatively low frequency of alcohol drinking, and the proportion who never drank was higher than that in most western European populations. It is possible that the deleterious effect of frequent alcohol drinking seen among the apolipoprotein e 4 carriers in our study is visible because of the typical binge drinking pattern of alcohol consumption (large quantities at a single session) in the population. Other types of drinking (that is, more frequently but not as much in any single session) may not have such detrimental effects even among e4 carriers.

With the possible exception of some Mediterranean countries, the harmful binge drinking pattern is common worldwide ${ }^{35}$ and the historically more healthy patterns of drinking in Europe are deteriorating in young people ${ }^{36}$ which underlines the importance of the health problems related to binge drinking. The acute adverse effects (such as injuries) of binge drinking are well known, but recent findings show that it also has harmful long term effects such as predisposition to atherosclerosis ${ }^{37}$ and coronary heart disease. ${ }^{38}$

\section{Strengths and limitations of study}

Our study design, a large population based prospective study with a long follow up time, increases the credibility of our findings. In studies with shorter follow up times and where alcohol consumption is assessed only in old age, subclinical dementia may affect consumption-but this kind of bias is unlikely in our study, where alcohol consumption was assessed at middle age.

The limitation of most studies of alcohol consumption is the reliability of the alcohol drinking data. However, errors in reporting of consumption are in general linearly related irrespective of how one tries to assess drinking, ${ }^{39}$ and so ranking of individuals into different alcohol drinking categories, as we did, should be possible. It was obvious that some of our participants drank more alcohol than they reported, but this would bias our results only if the difference between actual and reported alcohol drinking was dependent on participants' cognitive function. This might be so in analyses of alcohol drinking in old age-people with impaired cognitive abilities might forget how much they have drunk-but at the time of our midlife assessment most of our subjects were probably cognitively intact.

We investigated the possibility of selection bias in terms of non-participation. Non-participants were more likely to have dementia than the participants, but, since the non-participants did not differ significantly from the participants in their midlife alcohol consumption, their non-participation was not likely to have biased the associations between midlife alcohol drinking and mild cognitive impairment or dementia. There is also the possibility of selective survival related to apolipoprotein E polymorphism. Heavy acute loads of alcohol, which are known to increase vascular morbidity, ${ }^{37}$ more probably occurred in the frequent drinkers of alcohol than in the other groups. If the presence of the apolipoprotein e4 allele was related to increased mortality in this group, then our results would underestimate the relation between alcohol drinking and dementia.

\section{Conclusions}

Our results suggest that frequent alcohol drinking in middle age is associated with cognitive impairment in later life. For frequent drinking to be associated with increased risk of dementia, however, the presence of the apolipoprotein e4 allele may be necessary. This observation that apolipoprotein e4 may modify the effect of alcohol is in agreement with the concept that cognitive status is the consequence of both genetic and environmental factors. However, the mechanism by which moderate alcohol drinking could preserve cognitive function remains to be clarified. Is it alcohol as such or some other social and lifestyle factors that co-associate with certain drinking habits? Until all such factors and associations with cognitive functioning have been identified, we must be careful in how we interpret results relating to alcohol consumption. Our current data indicate that frequent alcohol drinking has harmful effects on the brain, and this may be more pronounced if there is genetic susceptibility. We therefore do not want to encourage people to drink more alcohol in the belief that they are protecting themselves against dementia.

We thank the colleagues in the CAIDE study group for their cooperation in data collection and management.

Contributors: MK and E-LH were the principal investigators in diagnosing dementia. TA analysed the data and drafted the paper. MK assisted in analyses and writing. MK, E-LH, AN, JT, and HS contributed to the conception and design of the study, JT and AN were involved in the baseline surveys for the study. TA, E-LH, MV, IK, LF, BW, HS, JT, AN and MK took part in planning the study and interpreting the data and commented on the manuscript. MK is the guarantor. 


\section{What is already known on this topic}

A J shaped or $\mathrm{U}$ shaped relation between alcohol consumption and cognitive impairment and dementia has been reported

The apolipoprotein e4 allele may modify the effect of alcohol on cognitive impairment and dementia, but the few studies conducted produced conflicting results

\section{What this study adds}

This is the first population based study that prospectively evaluated the effects of midlife alcohol drinking on the development of mild cognitive impairment and dementia in old age, taking into account genetic susceptibility

Midlife alcohol drinking had a U shaped association with mild cognitive impairment in old age, but no such association emerged in relation to dementia

Apolipoprotein E seemed to modify the association between alcohol consumption and dementia; the risk of dementia increased with increasing frequency of alcohol drinking among carriers of the e4 allele

Funding: Supported by the Aging Program of the Academy of Finland, EVO-grants of Kuopio University Hospital $(5772708,5772720)$ and Academy of Finland grants 103334 and 206951, SADF (Insamligsstiftelsen för Alzheimer- och Demensforskning), and the Gamla Tjänarinnor Foundation.

Competing interests: None declared.

Ethical approval: The ethics committees of the University of Kuopio and the Kuopio University Hospital, Kuopio, Finland, approved the study protocol.

1 Cervilla JA, Prince M, Mann A. Smoking, drinking and incident cognitive impairment a cohort community based study included in the Gospel Oak project. J Neurol Neurosurg Psychiatry 2000;68:622-6.

2 Launer LJ, Feskens EJ, Kalmijn S, Kromhout D. Smoking, drinking, and thinking. The Zutphen elderly study. Am J Epidemiol 1996;143:219-27.

3 Hébert LE, Scherr PA, Beckett LA, Albert MS, Rosner B, Taylor JO, et al. Relation of smoking and low-to-moderate alcohol consumption to change in cognitive function: longitudinal study in a defined community of older persons. Am J Epidemiol 1993;137:881-91.

4 Graves AB, van Duijn CM, Chandra V, Fratiglioni L, Heyman A, Jorm AF, et al. Alcoho and tobacco consumption as risk factors for Alzheimer's disease: a collaborative re-analysis of case-control studies. EURODEM Risk Factors Research Group. Int J Fpidemiol 1991;20(suppl 2):S48-57.

5 Hébert LE, Scherr PA, Beckett LA, Funkenstein HH, Albert MS, Chown MJ, et al. Relation of smoking and alcohol consumption to incident Alzheimer's disease. Am J Epidemiol 1992;135:347-55.

6 Saunders R, Copeland JRH, Dewey ME, Davidson IA, McWilliam C, Sharma V, et al. Heavy drinking as a risk factor for depression and dementia in elderly men. $\mathrm{Br} J$ Psychiatry 1991;159:213-6.

7 Thomas VS, Rockwood KJ. Alcohol abuse, cognitive impairment, and mortality among older people. J Am Geriatr Soc 2001;49:415-20.

8 Kalmijn S, van Boxtel MPJ, Verschuren MVM, Jolles J, Launer LJ. Cigarette smoking and alcohol consumption in relation to cognitive performance in middle age. Am J Epidemiol 2002;156:936-44.

9 Galanis DJ, Joseph C, Masaki KH, Petrovich H, Ross GW, White L. A longitudinal study of drinking and cognitive performance in elderly Japanese American men: the of drinking and cognitive performance in elderly Japanese

10 Leroi I, Sheppard J-M, Lyketsos CG. Cognitive function after 11.5 years of alcohol use: relation to alcohol use. Am J Epidemiol 2002;156:747-52.

11 Orgogozo JM, Dartigues JF, Lafont S, Letenneur L, Commenges D, Salamon R, et al. Wine consumption and dementia in the elderly: a prospective community study in the Bordeaux area. Rev Neurol (Paris) 1997;153:185-92.

12 Ruitenberg A, van Swieten JC, Wittemann JCM, Mehta KM, van Duijn CM, Hofman A, et al. Alcohol consumption and risk of dementia: the Rotterdam study. Lancel 2002;359:281-6.

13 Huang W, Qiu C, Winblad B, Fratiglioni L. Alcohol consumption and incidence of dementia in a community sample aged 75 years and older. J Clin Epidemiol 2002;55:959-64

14 Lindsay J, Laurin D, Verrault R, Hébert R, Helliwell B, Hill GB, et al. Risk factors for Alzheimer's disease: a prospective analysis from the Canadian study of health and aging. Am J Epidemiol 2002;156:445-53.

15 Carmelli D, Swan GE, Reed T, Schellenberg GD, Christian JC, Gaziano J, et al. The effect of apolipoprotein $\mathrm{E}$ epsilon4 in the relationships of smoking and drinking to cognitive function. Neuroepidemiology 1999;18:125-33.
16 Dufouil C, Tzourio C, Brayne C, Berr C, Amouyel P, Alpérovitch A. Influence of apolipoprotein $\mathrm{E}$ genotype on the risk of cognitive deterioration in moderate drinkers and smokers. Epidemiology 2000;11:280-4.

17 Mukamal KJ, Kuller LH, Fitzpatrick AL, Longstreth WT Jr, Mittleman MA, Soscovick DS. Prospective study of alcohol consumption and risk of dementia in older adults. JAMA 2003;289:1405-13.

18 Petersen RC, Doody R, Kurz A, Mohs RC, Morris JC, Rabins PV, et al. Current concepts in mild cognitive impairment. Arch Neurol 2001;58:1985-92.

19 Petersen RC, Smith GE, Waring SC, Ivnik RJ, Tangalos EG, Kokmen E. Mild cognitive impairment: clinical characterization and outcome. Arch Neurol 1999;56:303-8.

20 Larrieu S, Letenneur L, Orgogozo JM, Fabrigoule C, Amieva H, Le Carret N, et al. Incidence and outcome of mild cognitive impairment in a population-based prospective dence and outcome of mild cognitive
cohort. Neurology 2002;59:1594-9.

21 Braak E, Griffing K, Arai K, Bohl J, Bratzke H, Braak H. Neuropathology of Alzheimer's disease: what is new since A. Alzheimer? Eur Arch Psychiatry Clin Neurosci 1999;249(suppl 3):14-22.

22 Kivipelto M, Helkala EL, Hänninen T, Laakso MP, Hallikainen M, Alhainen K, et al. Midlife vascular risk factors and late-life mild cognitive impairment. A populationbased study. Neurology 2001;56:1683-9.

23 Puska P, Tuomilehto J, Salonen J, Neittaanmäki L, Mäki J, Virtamo J, et al. Changes in coronary risk factors during comprehensive five-year community programme to control cardiovascular diseases (North Karelia project). BMJ 1979:2;1173-8.

24 Puska P, Salonen JT, Nissinen A, Tuomilehto J, Vartiainen E, Korhonen H, et al. Change in risk factors for coronary heart disease during 10 years of a community intervention in risk factors for coronary heart disease during 10 years of a co
programme (the North Karelia project). BMJ 1983;287:1840-4.

25 Vartiainen E, Puska P, Jousilahti P, Korhonen HJ, Tuomilehto J, Nissinen A. Twenty-years trends in coronary risk factors in North-Karelia and in other areas of Finland. Int J Epidemiol 1994;23:495-504

26 Tsukamoto K, Watanabe T, Matsushima T, Kinoshita M, Kato H, Hashimoto Y, et al. Determination by PCR-RFLP of ApoE genotype in a Japanese population.J Lab Clin Med 1993;121:598-602.

27 Folstein MF, Folstein SE, McHugh PR. "Mini-mental state": a practical method for grading the cognitive state of patients for the clinician. J Psychiatr Res 1975;12:189-98.

28 Petersen RC, Smith GE, Ivnik RJ, Tangalos EG, Schaid DJ, Thibodeau SN, et al. Apolipoprotein E status as a predictor of development of Alzheimer's disease in memory-impaired individuals. JAMA 1995;273:1274-8.

29 Smith GE, Petersen RC, Parisi JE, Ivnik RJ, Kokmen E, Tangalos EG, et al. Definition, course and outcome of mild cognitive impairment. Aging Neuropsychol Cogn 996:3:141-7.

30 McKhann G, Drachman D, Folstein M, Katzman R, Price D, Stadlan EM. Clinical diagnosis of Alzheimer's disease: report of the NINCDS-ADRDA Work Group under the auspices of Department of Health and Human Services Task Force on Alzheimer's Disease. Neurology 1984;34:939-44.

31 Agarwal DP. Cardioprotective effects of light-moderate consumption of alcohol: a review of putative mechanisms. Alcohol Alcohol 2002;37:409-15.

32 Commenges D, Scotet V, Renaud S, Jacqmin-Gadda H, Barberger-Gateau P, Dartigues JF. Intake of flavonoids and risk of dementia. Eur J Epidemiol 2000;16:357-63.

33 Truelsen T, Thudium D, Grønbæk M. Amount and type of alcohol and risk of dementia: the Copenhagen city heart study. Neurology 2002;59:1313-9.

34 Mahley RW, Rall SC Jr. Apolipoprotein E: far more than a lipid transport protein. Annu Rev Genomics Hum Genet 2000;1:507-37.

35 Rehm J, Rehn N, Room R, Monteiro M, Gmel G, Jernigan D, et al. The global distrbution of average volume of alcohol consumption and patterns of drinking. Eur Addict Res 2003;9:147-56.

36 Hibell B, Andersson B, Ahlström S, Balakireva O, Jarnasson T, Kokkevi A, et al. The 1999 ESPAD report. Alcohol and other drug use among students in 30 European countries. Stockholm, Pompidou Group, Council of Europe, 2000.

37 Kauhanen J, Kaplan GA, Goldberg DE, Salonen R, Salonen JT. Pattern of alcohol drinking and progression of atherosclerosis. Arterioscler Thromb Vasc Biol 1999;19:3001-6.

38 Rehm J, Room R, Graham K, Monteiro M, Gmel G, Sempos CT. The relationship of average volume of alcohol consumption and patterns of drinking to burden of disease: an overview. Addiction 2003;98:1209-28.

39 Feunekes GI, van't Veer P, van Staveren WA, Kok FJ. Alcohol intake assessment: the sober facts. Am J Epidemiol 1999;150:105-12.

(Accepted 6 July 2004)

doi 10.1136/bmj.38181.418958.BE

Aging Research Center, Division of Geriatric Epidemiology, Neurotec, Karolinska Institutet, Box 6401, 11382 Stockholm, Sweden

Tiia Anttila PhD student

Ingemar Kåreholt statistician

Laura Fratiglioni professor

Miia Kivipelto researcher

Department of Public Health and General Practice, University of Kuopio, Box 1627, 70211 Kuopio, Finland

Eeva-Liisa Helkala neuropsychologist

Division of Geriatric Medicine, Neurotec, Karolinska Institutet, Karolinska

University Hospital, Huddinge, 14186 Stockholm

Matti Viitanen professor

Bengt Winblad professor

Department of Neuroscience and Neurology, University of Kuopio, Kuopio Hilkka Soininen professor

Department of Epidemiology and Health Promotion, National Public Health Institute, Mannerheimintie 166, 00300 Helsinki, Finland

Jaakko Tuomilehto professor

Aulikki Nissinen professor

Correspondence to: Miia Kivipelto Miia.Kivipelto@uku.fi 